\title{
Evidence of Contextual Fear after Lesions of the Hippocampus: A Disruption of Freezing But Not Fear-Potentiated Startle
}

\author{
Kenneth A. McNish, Jonathan C. Gewirtz, and Michael Davis \\ Departments of Psychiatry and Psychology, Yale University School of Medicine, Connecticut Mental Health Center, New \\ Haven, Connecticut 06508
}

\begin{abstract}
The roles of the dorsal hippocampus and the central nucleus of the amygdala in the expression of contextual fear were assessed using two measures of conditioned fear: freezing and fear-potentiated startle. A discriminable context conditioning paradigm was developed that demonstrated both conditioned freezing and fear-potentiated startle in a context paired previously with foot shock, relative to a context in which foot shock had never been presented. Post-training lesions of the central nucleus of the amygdala completely blocked both contextual
\end{abstract}

freezing and fear-potentiated startle. Post-training lesions of the dorsal hippocampus attenuated contextual freezing, consistent with previous reports in the literature; however, these same lesions had no effect on fear-potentiated startle, suggesting preserved contextual fear. These results suggest that lesions of the hippocampus disrupt the freezing response but not contextual fear itself.

Key words: context; fear; conditioning; hippocampus; amygdala; freezing; startle; learning; memory
It is commonly believed that contextual fear conditioning, like spatial learning, is a hippocampal-dependent task. The primary evidence supporting this conclusion has come from studies that evaluated the effects of amygdala or hippocampal lesions on freezing elicited by either contextual or explicit cues (Kim and Fanselow, 1992; Phillips and LeDoux, 1992). In these studies, an explicit cue was paired with shock in a distinctive context. After training, fear conditioning to the context was assessed by the amount of freezing the animals displayed when returned to the context in which they were trained. Fear to the explicit cue was assessed by the amount of freezing elicited by the cue when presented in a neutral context. Amygdala lesions blocked freezing to both the context and the explicit cue, consistent with the notion that this structure is critically involved in mediating conditioned fear responses (Kapp et al., 1982,1984; Davis, 1992; LeDoux, 1992). In contrast, hippocampal lesions made either before training (Phillips and LeDoux, 1992) or after training (Kim and Fanselow, 1992) disrupted conditioned freezing to the context but not to the explicit cue. The most common interpretation of these results has been that the hippocampal lesions disrupted the ability of the animal to form complex, polymodal associations, as would be required in forming a representation of context.

There are, however, alternative explanations for these results. First, based on principles of associative learning, one would expect stronger conditioning to the explicit cues present on a conditioning trial than to the experimental context (Wagner, 1981). Thus, hippocampal lesions may preferentially disrupt weak

Received July 1, 1997; revised Sept. 12, 1997; accepted Sept. 17, 1997.

This work was supported by National Institute of Mental Health Grants $\mathrm{MH}$ 47840, MH-19951, and MH-11370, Research Scientist Development Award MH00004, and Air Force Office of Scientific Research Grant F49620. We thank Jeansok Kim for his valuable discussions on context conditioning and the role of the hippocampus and Diane Lendroth for her assistance with histology. We also thank Ed and Lori Adams for the use of Bear Island, where portions of this manuscript were prepared.

Correspondence should be addressed to Michael Davis, Yale University School of Medicine, Departments of Psychiatry and Psychology, Connecticut Mental Health Center, 34 Park Street, New Haven, CT 06508.

Copyright (C) 1997 Society for Neuroscience $0270-6474 / 97 / 170001-08 \$ 05.00 / 0$ versus strong memories. Second, lesions of the hippocampus have been shown to increase activity in an open field (Roberts et al., 1962; Teitelbaum and Milner, 1963; Douglas and Isaacson, 1964; Blanchard et al., 1977; Diaz-Granados et al., 1994; Maren and Fanselow, 1997), which might interfere with freezing. In fact, Douglas (1967) proposed that hippocampal lesions produce a deficit in behavioral inhibition, of which freezing is an example. The experimental designs of both Kim and Fanselow (1992) and Phillips and LeDoux (1992) controlled for any simple performance effects on freezing by measuring freezing to the explicit cue. If there had been a simple performance effect on freezing, they should have detected this as a decrease in freezing to the explicit cue as well as to the context. However, it is also possible that a performance effect on activity could interact with the strength of the conditioned response (Good and Honey, 1997). Thus, hippocampal lesions could preferentially disrupt weak conditioned freezing responses to the context but not strong conditioned freezing responses to the explicit cue. In fact, Phillips and LeDoux (1994) subsequently reported that pretraining hippocampal lesions disrupted freezing to a context when an explicit cue was paired with shock in that context but had no effect when a context was paired directly with shock. This finding is consistent with the weak versus strong hypothesis, because one would expect stronger conditioned freezing to the context in the latter case than in the former.

The present studies were designed to evaluate the effects of hippocampal lesions on contextual fear using a measure of conditioned fear other than freezing. The acoustic startle reflex has proven useful in evaluating fear to explicit cues. The startle reflex can be reliably increased when elicited in the presence of an explicit cue that has been paired with shock (fear-potentiated startle; Brown et al., 1951; Davis, 1986). Electrolytic or chemical lesions of the amygdala block the expression of fear-potentiated startle to an explicit cue (Hitchcock and Davis, 1986; Sananes and Davis, 1992; Campeau and Davis, 1995). It has also been demonstrated that the startle reflex can be increased when a context, as opposed to an explicit cue, is used as the conditioned stimulus 
(Campeau et al., 1991). The aim of the present studies was to develop a paradigm that produced discriminable context conditioning using both freezing and fear-potentiated startle as measures of contextual fear. Subsequently, we evaluated the effects of lesions of the central nucleus of the amygdala on the expression of contextual fear with the prediction that the lesions would block the expression of both conditioned freezing and fear-potentiated startle. We next evaluated the effects of dorsal hippocampal of lesions on the expression of contextual fear. We presumed that the hippocampal lesions would disrupt contextual freezing, as has been reported previously. If the lesions also disrupted fearpotentiated startle, this would be consistent with the idea that the hippocampus is critical for contextual fear conditioning. However, if the lesions disrupted freezing but had no effect on fearpotentiated startle, it would suggest that the context still elicited the conditioned response of fear.

\section{MATERIALS AND METHODS}

Animals. A total of 100 male albino Sprague Dawley rats (Charles River Co., Portage, MI) weighing between 300 and 400 gm were used. All rats were housed in groups of five in hanging wire cages. The rats were maintained on a $12 \mathrm{hr}$ light/dark cycle (lights on at 7:00 A.M.) with food and water continuously available.

Apparatus. Two distinct chambers, A and B, were used. In chamber A, activity and startle testing were conducted in five identical stabilimeter devices that have been described previously (Cassella and Davis, 1986). Briefly, each stabilimeter consisted of an $8 \times 15 \times 15 \mathrm{~cm}$ Plexiglas and wire mesh cage suspended between compression springs within a steel frame. The floor of each stabilimeter consisted of four 6.0-mm-diameter stainless steel bars spaced $18 \mathrm{~mm}$ apart through which shock could be administered. Cage movement resulted in displacement of an accelerometer by which the resultant voltage was proportional to the velocity of the cage displacement. The analog output of the accelerometer was amplified and digitized on a scale of $0-4096$ units by a MacADIOS II board (GW instruments, Somerville, MA) interfaced to a Macintosh II microcomputer.

Each stabilimeter was located within a $68.5 \times 35.5 \times 42 \mathrm{~cm}$ ventilated plywood isolation box. This inner isolation box was located within an additional outer $76 \times 47 \times 51 \mathrm{~cm}$ ventilated plywood isolation box. This "double housing" of stabilimeter devices was used to prevent ultrasonic communication among the rats. All five stabilimeter and double housing isolation boxes were located in a ventilated, sound-attenuating chamber $(2.5 \times 2.5 \times 2 \mathrm{~m}$; Industrial Acoustics, Bronx, NY $)$. A surveillance camera (model ITC-40; Ikegami, Utsunomiya, Japan) was positioned behind each stabilimeter within the inner isolation box and connected to a television monitor located outside of the Industrial Acoustics isolation chamber. A red light bulb $(7.5 \mathrm{~W})$ was located on the floor of the inner isolation box to provide illumination for the cameras in the otherwise dark box.

Background noise $(0-20 \mathrm{kHz}, 55 \mathrm{~dB})$ was produced by a white noise generator (Lafayette model 15800) and delivered through highfrequency speakers (Radio Shack Supertweeters; range, 5-40 kHz) located $2 \mathrm{~cm}$ from the front of each stabilimeter. Ventilation fans attached to the side walls of both the inner and outer isolation boxes produced some additional background noise that raised the overall ambient background noise to $65 \mathrm{~dB}$. The startle stimulus was either a 100,105 , or 110 $\mathrm{dB}, 50 \mathrm{msec}$ burst of white noise generated by a white noise generator (Lafayette, model 15800) and delivered through the same speakers as the background noise. Sound pressure level measurements were made with a Bruel \& Kjaer model 2235 sound level meter (A scale; random input). The foot shock was produced by five LeHigh Valley shock generators (SGS-004; LeHigh Valley, Beltsville, MD) located outside the sound attenuating chamber. Shock intensity was measured with a $1 \mathrm{k} \Omega$ resistor across a differential channel of an oscilloscope in series with a $100 \mathrm{k} \Omega$ resistor connected between adjacent floor bars within each stabilimeter. Current was defined as the root mean square voltage across the $1 \mathrm{k} \Omega$ resistor where $\mathrm{mA}=0.707 \times 0.5 \times$ peak-to-peak voltage. According to this method, the shock intensity was $0.6 \mathrm{~mA}$ with a duration of $500 \mathrm{msec}$. The presentation and sequencing of all stimuli were under the control of a Macintosh II microcomputer.

Chamber $\mathrm{B}$ differed from chamber $\mathrm{A}$ in terms of location, odor, somatosensory cues, and isolation of the animals. A distinctive route to chamber B was taken to minimize similarities in the transport of the animals. Each cage was cleaned with a $1 \%$ acetic acid solution between use (compared with water only in chamber A) to provide a distinctive olfactory environment. Two chains, $9 \mathrm{~cm}$ in length, were hung from the ceiling of each cage to provide a distinctive somatosensory environment. The individual stabilimeters were not isolated from one another as in chamber A. The background noise, $55 \mathrm{~dB}$, was delivered through a separate Jamocar 70 speaker (range, $0.02-20 \mathrm{kHz}$ ) located $\sim 70 \mathrm{~cm}$ in front of each cage. A single ventilation fan was attached to the outside of the sound-attenuating chamber $(2.5 \times 2.5 \times 2 \mathrm{~m}$; Industrial Acoustics $)$ and did not raise the ambient background noise to $>55 \mathrm{~dB}$. In all other respects, chamber B was similar to chamber A.

Surgery. Rats were anesthetized with sodium pentobarbital $(50 \mathrm{mg} / \mathrm{kg}$, i.p.) and placed in a Kopf stereotaxic instrument. Post-training electrolytic lesions of the central nucleus of the amygdala were performed by passing a $0.1 \mathrm{~mA}$ anodal current for $90 \mathrm{sec}$ at the following coordinates: anteroposterior (AP), $-1.9,-3.0$; mediolateral (ML), $\pm 4.0,4.4$; and dorsoventral (DV), -8.6, -8.4. Post-training electrolytic lesions of the dorsal hippocampus were performed by passing a $1.0 \mathrm{~mA}$ anodal current for $15 \mathrm{sec}$ at the following coordinates: AP, $-3.0,-4.0,-5.0$; ML, \pm 1.8 , 3.0, 3.0; and DV, $-4.0,-4.0,-4.0$. The coordinates used for the dorsal hippocampal lesions were consistent with studies previously demonstrating behavioral effects of these lesions (e.g., Kim and Fanselow, 1992). Sham-lesioned animals were treated identically, except that no current was passed. All subjects were allowed $7-10 \mathrm{~d}$ recovery from surgery before training or testing.

Discriminable context conditioning. Forty animals received two identical pretraining test sessions. Half of the animals were tested in chamber A, the other half in chamber B. In each test session, baseline activity was sampled once every $10 \mathrm{sec}$ for $5 \mathrm{~min}$. After activity sampling, animals received a total of 30 startle stimuli at three different intensities $(100,105$, and $110 \mathrm{~dB}$ ) at an interstimulus interval of $30 \mathrm{sec}$. Each test session was $20 \mathrm{~min}$ in duration. Animals were matched into two equivalent groups, same $(n=20)$ or different $(n=20)$, based on their baseline activity and startle responding on the second day of testing. Animals in the same group $(\mathrm{A}-\mathrm{A}$ and $\mathrm{B}-\mathrm{B})$ were trained in the same chamber in which they were tested, whereas animals in the different group (A-B and B-A) were trained in a different chamber. The assignment of animals to groups and chambers was counterbalanced so that 10 animals were represented in each of the four possible training and testing conditions. Two days of training were given. On each day, baseline activity was sampled once every $10 \mathrm{sec}$ for $5 \mathrm{~min}$ before the administration of shocks. After activity sampling, animals received $100.6 \mathrm{~mA}$ unsignaled foot shocks at a $2 \mathrm{~min}$ variable interstimulus interval (range, $1-3 \mathrm{~min}$ ). Each training session was $25 \mathrm{~min}$ in duration. Forty-eight hours after training, animals received a post-training test that was identical to the pretraining test.

Amygdala lesions. Training and testing were identical to the procedures detailed above, except that all training and testing took place in chamber A. Twenty animals were matched into two equivalent groups based on their baseline activity and startle responding during pretraining testing and their baseline activity and reactivity to the foot shock administered during training. Surgery was performed $24-48 \mathrm{hr}$ after training and $7-8$ $\mathrm{d}$ before the final test.

Hippocampal lesions. Training and testing were identical to the procedures used to evaluate post-training lesions of the central nucleus of the amygdala. Two replications were conducted with 20 animals in each replication.

Behavioral measures. The output of the accelerometer was used to measure baseline activity, startle amplitude, and shock reactivity. An activity sample was defined as the peak accelerometer voltage that occurred during a 500-msec sampling period. Startle amplitude was defined as the peak accelerometer voltage that occurred during the first $200 \mathrm{msec}$ after onset of the startle stimulus. Shock reactivity was measured as the peak accelerometer voltage during the $500 \mathrm{msec}$ presentation of the shock.

Freezing was defined as the mean activity before training minus the mean activity after training. This measure of freezing has been shown to be highly correlated with observational measures of freezing and can reliably detect different magnitudes of freezing (Leaton and Borszcz, 1985; Gewirtz et al., 1997). Fear-potentiated startle was defined as the mean startle amplitude after training minus the mean startle amplitude before training.

Histology. At the end of each experiment, rats were overdosed with chloral hydrate and perfused intracardially first with saline and then with 

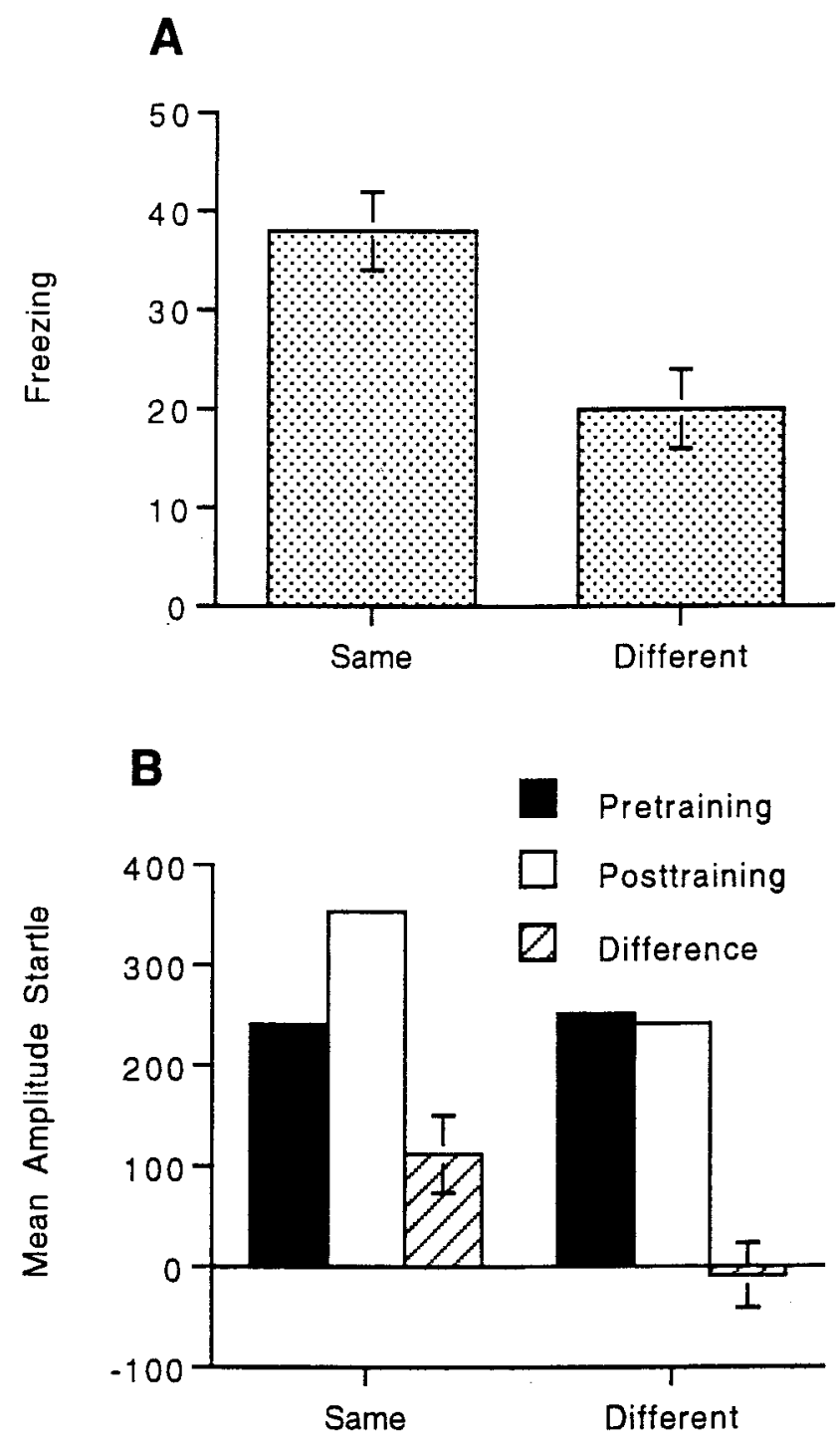

Figure 1. $A$, Mean freezing (pretraining activity minus post-training activity) and SEMs for animals trained and tested in the same versus different contexts. $B$, Mean amplitude startle response, pretraining, posttraining, difference scores (post-training minus pretraining), and SEMs for animals trained and tested in the same versus different contexts.

$10 \%$ buffered formalin phosphate (Fisher Scientific, Houston, TX). Brains remained in a $30 \%$ sucrose/formalin solution $(30 \mathrm{gm}$ sucrose $/ 70 \mathrm{ml}$ formalin) for at least $48 \mathrm{hr}$ before sectioning. Forty micrometer coronal sections were taken from lesioned animals. Every third section was mounted and stained with cresyl violet to evaluate the extent of the lesions.

\section{RESULTS}

\section{Discriminable context conditioning}

Figure $1 A$ displays the mean freezing (pretraining activity minus post-training activity) for animals trained and tested in the same versus different contexts. Both groups froze during testing, indicating fear to the context. However, animals trained and tested in the same context froze approximately twice as much as animals trained and tested in different contexts. The contextual freezing displayed by the different group is likely to reflect generalization between the training and testing chambers, because the two chambers shared a number of similar features. To analyze the data, the mean activity over the 5 min sampling period before and after training was computed for each animal. A preliminary ANOVA did not reveal any main effects of replication (1 and 2) or chamber (A and B), hence the data were combined over replication and chamber for all subsequent analyses. An ANOVA using group (same vs different) as a between-subjects factor and session (pretraining vs post-training) as a within-subjects factor revealed a main effect of session, $F_{(1,38)}=97.49 ; p<0.001$, indicating significant freezing in both the same and different groups. There was also a significant group by session interaction, $F_{(1,38)}=9.85 ; p<0.003$, indicating more freezing in the same versus different group. Subsequent paired $t$ tests revealed significant freezing in both the same, $t_{(19)}=8.92, p<0.001$, and different groups, $t_{(19)}=4.92 ; p<0.001$.

Figure $1 B$ displays the mean amplitude startle before and after training as well as the difference scores for the same and different groups. There was an increase in startle responding in animals trained and tested in the same context, indicating contextual fear; however, there was no increase in animals trained and tested in different chambers. Interestingly, there was no evidence of generalization across chambers on the startle measure as there was with the freezing measure. This suggests that the freezing response may be more sensitive to lower levels of fear than the startle reflex. To analyze the data, the mean amplitude startle before and after training was computed for each animal. A preliminary ANOVA revealed a main effect of replication, $F_{(1,32)}$ $=4.91 ; p<0.03$, indicating greater overall startle responding in the second replication compared with the first. There was also a main effect of chamber, $F_{(1,32)}=22.90 ; p<0.001$, indicating greater overall startle responding in chamber A compared with chamber B. However, there were no group by replication or group by chamber interactions; therefore, the data were combined over replication and chamber for all subsequent analyses. An ANOVA using group (same vs different) and session (pretraining vs posttraining) as factors revealed a main effect of session, $F_{(1,38)}=$ 4.27; $p<0.046$, indicating greater mean startle amplitudes after training relative to before training. More importantly, there was a significant group by session interaction, $F_{(1,38)}=5.97 ; p<0.019$, indicating a greater increase in startle in the same group relative to the different group. Subsequent paired $t$ tests revealed significant fear-potentiated startle in the same group, $t_{(19)}=2.92 ; p<$ 0.009 , but not in the different group, $t_{(19)}=-0.30$.

An analysis of the freezing and shock reactivity data from the training sessions was performed. The mean freezing (pretraining activity minus activity on the second $d$ of training) and SEM for each group were as follows: same, $35 \pm 5$; and different, $31 \pm 5$. An ANOVA using group (same vs different) and session (pretraining minus second day of training) as factors revealed a main effect of session, $F_{(1,38)}=100.34 ; p<0.001$, indicating significant freezing across groups but no group by session interaction, $F_{(1,38)}$ $=0.40$, indicating that the amount of freezing during training did not differ by group. Thus, the same and different groups showed equivalent levels of freezing to the contexts in which they were trained. The mean shock reactivity for each animal was computed for the 10 foot shocks presented on each of the $2 \mathrm{~d}$ of training. The mean shock reactivity \pm SEM for each group were as follows: same, $904 \pm 90$; and different, $866 \pm 92$. A one-way ANOVA using group as a factor yielded no main effect of group, $F_{(1,38)}=0.09$, indicating that differences in shock reactivity during training could not account for the differences in contextual freezing and fear-potentiated startle between groups. 
A
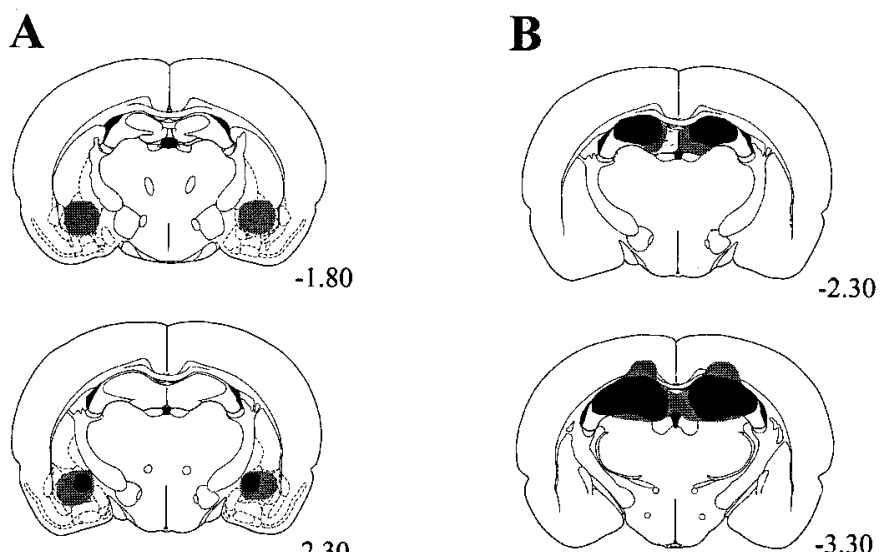

$-2.30$

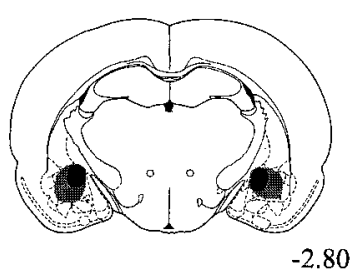

$-2.80$

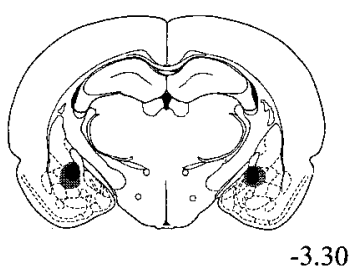

$-3.30$
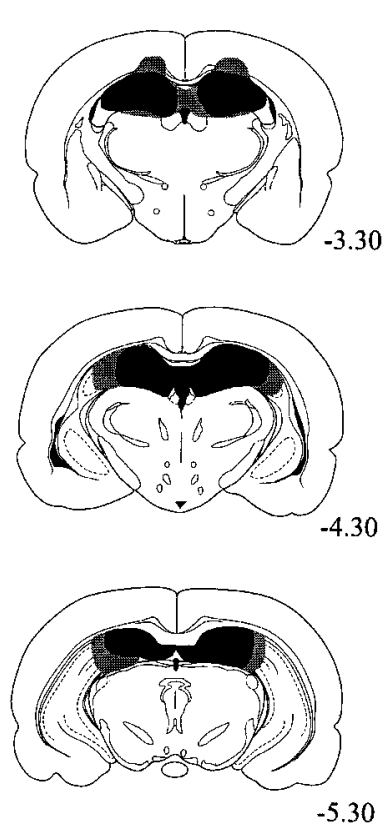

$-5.30$
Figure 2. Serial reconstructions of the smallest (black) and largest (gray) lesions for lesions of the central nucleus of the amygdala $(A)$ and lesions of the dorsal hippocampus $(B)$. The numbers below each section represent the anterior-posterior coordinates relative to bregma. Adapted from Paxinos and Watson (1997).

\section{Amygdala lesions}

Figure $2 A$ displays serial reconstructions of the smallest (black) and largest (gray) lesions of the central nucleus of the amygdala (CeA). Three of the animals in the amygdala group were omitted from the statistical analyses because of significant sparing of the CeA. This left seven animals in the amygdala group and 10 animals in the sham group. All remaining amygdala-lesioned animals sustained significant damage to the medial $\mathrm{CeA}$. The smallest lesions spared the most anterior aspects of the CeA and some of the lateral CeA. The largest lesions also damaged the basolateral nucleus of the amygdala.

Figure $3 A$ displays the mean freezing for animals that received either sham lesions or electrolytic lesions of the central nucleus of the amygdala. Sham-lesioned animals showed robust contextual freezing that was blocked completely by lesions of the amygdala. The data were analyzed as in the previous experiment. An ANOVA using group (sham vs amygdala) and session (pretraining vs post-training) as factors revealed a main effect of group, $F_{(1,15)}=9.50 ; p<0.008$, a main effect of session, $F_{(1,15)}=20.47$; $p<0.001$, and a significant group by session interaction, $F_{(1,15)}=$ $17.75 ; p<0.001$, indicating more freezing in the sham than amygdala group. Subsequent paired $t$ tests revealed significant freezing in the sham group, $t_{(9)}=6.68 ; p<0.001$, but not in the amygdala group $t_{(6)}=0.21$.

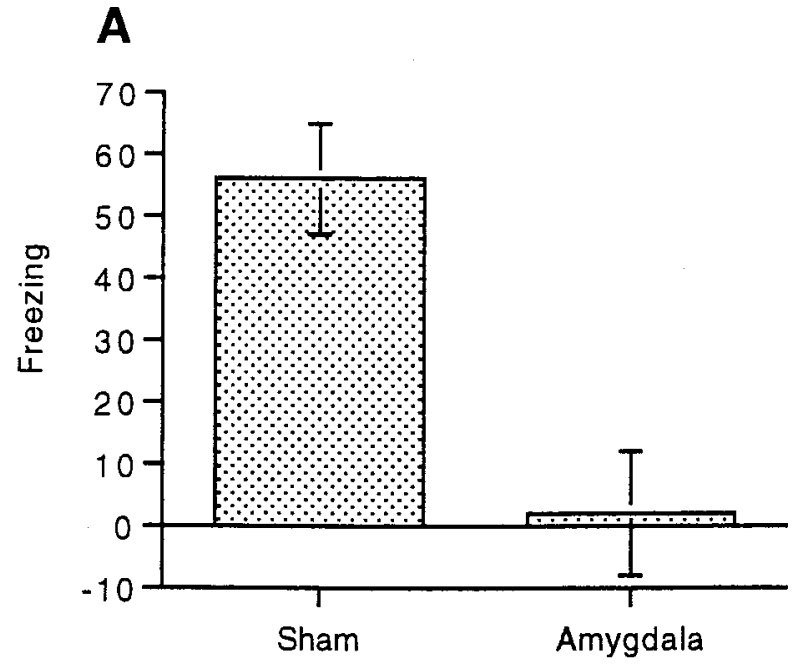

\section{B}

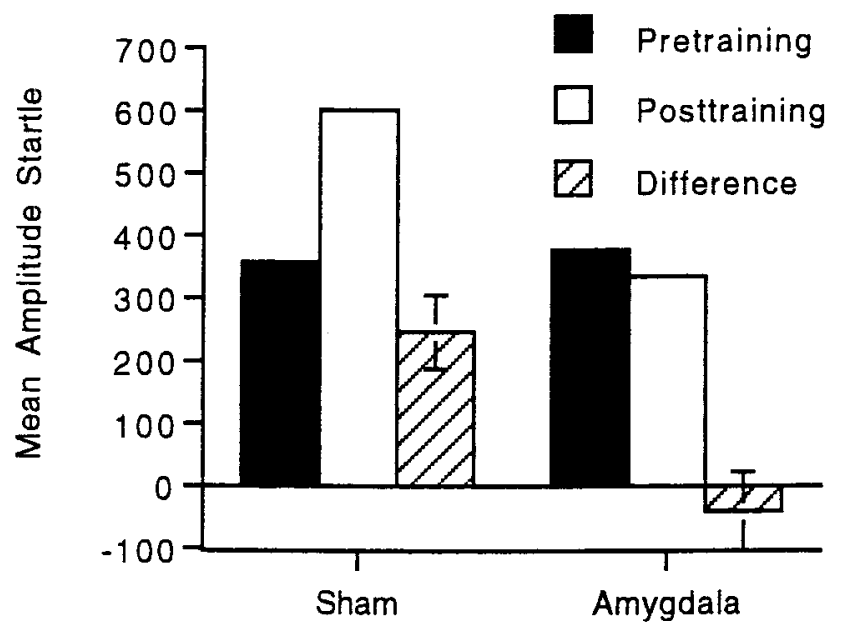

Figure 3. A, Mean freezing (pretraining activity minus post-training activity) and SEMs for animals that received either sham lesions or electrolytic lesions of the central nucleus of the amygdala. $B$, Mean amplitude startle response, pretraining, post-training, difference scores (post-training minus pretraining), and SEMs for animals that received either sham lesions or electrolytic lesions of the central nucleus of the amygdala.

Figure $3 B$ displays the mean amplitude startle before and after training as well as the difference scores for the sham and amygdala groups. There was an increase in startle after training in the sham group that was completely blocked by lesions of the amygdala. An ANOVA on the startle data using the same factors as above revealed a main effect of session, $F_{(1,15)}=6.10 ; p<0.026$, indicating greater mean startle amplitudes after training relative to before training. More importantly, there was a significant group by session interaction, $F_{(1,15)}=12.07 ; p<0.003$, indicating greater fear-potentiated startle in the sham group than the amygdala group. Subsequent paired $t$ tests revealed significant fearpotentiated startle in the sham group, $t_{(9)}=4.41 ; p<0.002$, but not in the amygdala group, $t_{(6)}=-0.71$.

An analysis of the data from the training sessions was performed as in the previous experiment. The data of one animal 
from the sham group were excluded because of an error in recording shock reactivity on the second day of training. The mean freezing and SEM for each group were as follows: sham, $54 \pm 8$; and amygdala, $54 \pm 7$. The mean shock reactivity and SEM for each group were as follows: sham, $979 \pm 35$; and amygdala, $1099 \pm 131$. Thus differences in shock reactivity or contextual freezing during training cannot account for the differences in contextual freezing or fear-potentiated startle between the groups.

\section{Hippocampal lesions}

Figure $2 B$ displays serial reconstructions of the smallest (black) and largest (gray) lesions of the dorsal hippocampus. Three animals in the hippocampal group died after surgery. In addition, two of the animals in the hippocampal group were omitted from the statistical analyses because of significant damage to the overlying cortex, and one animal was omitted because of sparing of the anterior dorsal hippocampus. This left 14 animals in the hippocampal group and 20 animals in the sham group. All remaining hippocampal-lesioned animals sustained damage to the dentate gyrus and CA1, CA2, and CA3 fields. Smaller lesions spared the most anterior aspects of the dentate gyrus and the CA3 field. Larger lesions included minor damage to the overlying cortex as well as the most dorsal aspects of the thalamus.

Figure $4 A$ displays the mean freezing for animals that received either sham lesions or electrolytic lesions of the dorsal hippocampus. Hippocampal lesions produced an attenuation in contextual freezing relative to the sham-operated controls. The data were analyzed as in the previous experiments. A preliminary ANOVA revealed no effect of replication, so the data were combined for all subsequent analyses. An ANOVA using group (sham vs hippocampus) and session (pretraining vs post-training) as factors revealed a main effect of group, $F_{(1,32)}=4.66 ; p<0.038$, a main effect of session, $F_{(1,32)}=69.55 ; p<0.001$, and a significant group by session interaction, $F_{(1,32)}=5.81 ; p<0.022$, indicating an attenuation in freezing in the hippocampal lesioned group relative to the sham group. Subsequent paired $t$ tests revealed significant freezing in both the sham, $t_{(19)}=9.91 ; p<0.001$, as well as the hippocampal group, $t_{(13)}=3.25 ; p<0.006$.

Figure $4 B$ displays the mean amplitude startle before and after training as well as the difference scores for the sham and hippocampal groups. In contrast to the freezing data, lesions of the dorsal hippocampus had no effect on fear-potentiated startle. Thus, fear-potentiated startle, which was shown to be contextspecific in Experiment 1 and amygdala-dependent in Experiment 2, was preserved in hippocampal-lesioned animals, although these same animals showed an attenuation in freezing. An ANOVA on the startle data using the same factors as above revealed a main effect of session, $F_{(1,32)}=46.96 ; p<0.001$, indicating greater mean startle amplitudes after training relative to before. However, there was no group by session interaction, $F_{(1,32)}=0.02$, indicating that the magnitude of fear-potentiated startle did not differ between groups. To analyze the time course of extinction over testing, the startle data were combined over three trial blocks (data not shown). Repeating the above ANOVA adding block $1-10$ as a factor yielded a significant main effect of block, $F_{(9,288)}$ $=19.06 ; p<0.001$, and block by session interaction, $F_{(9,288)}=$ 3.31; $p<0.001$, indicating that fear-potentiated startle decreased over the course of testing. The extinction of fear-potentiated startle did not differ by group, $F_{(9,288)}=1.57$.

An analysis of the data from the training sessions was performed as in the above experiments. The data of one animal from
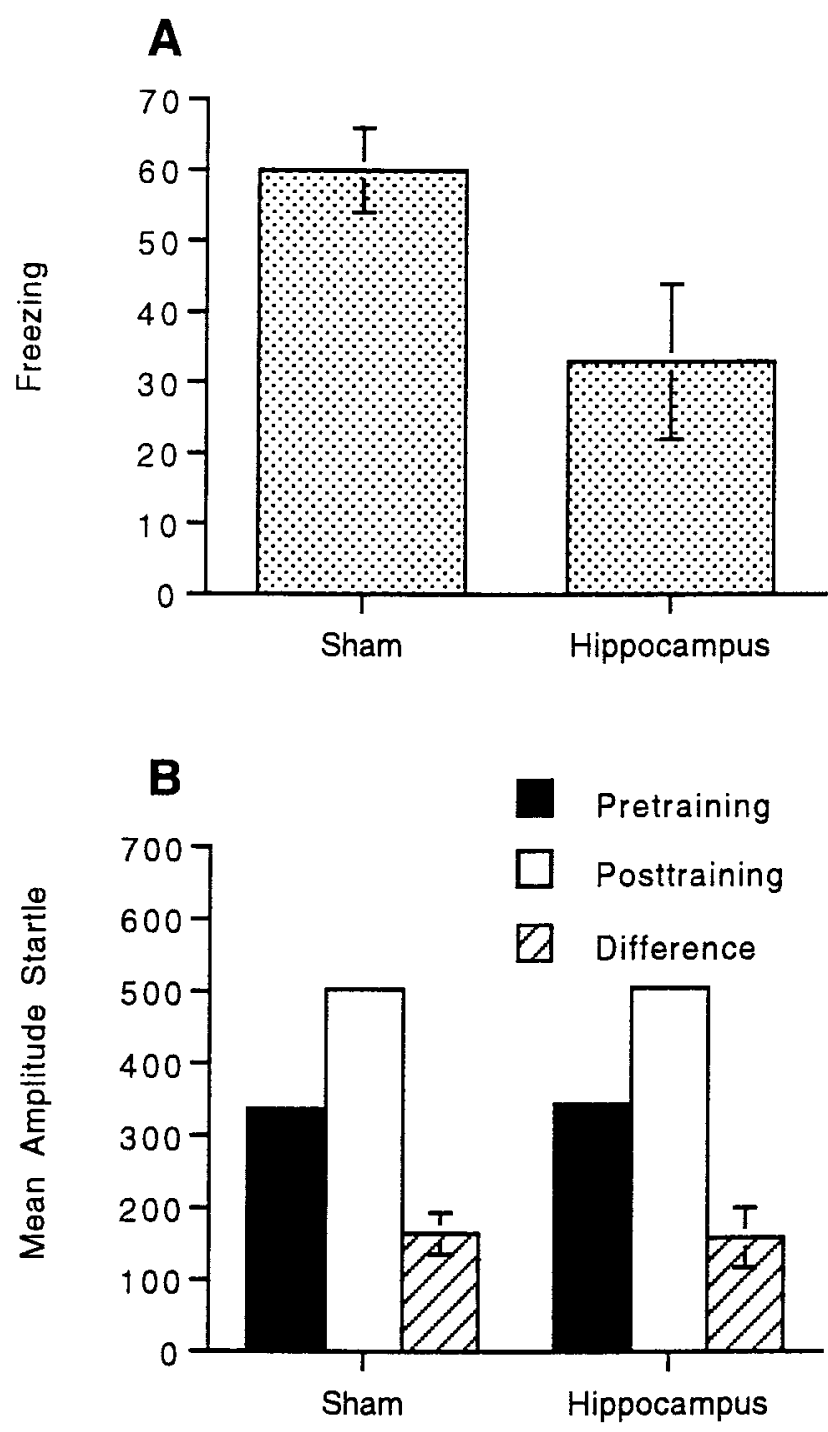

Figure 4. A, Mean freezing (pretraining activity minus post-training activity) and SEMs for animals that received either sham lesions or post-training lesions of the dorsal hippocampus. $B$, Mean amplitude startle response, pretraining, post-training, difference scores (posttraining minus pretraining), and SEMs for animals that received either sham lesions or post-training lesions of the dorsal hippocampus.

the sham group, as well as that of one animal from the lesion group, were excluded because of an error in recording shock reactivity on the second day of training. The mean freezing and SEM for each group during training were as follows: sham, $55 \pm$ 7; and hippocampus, $57 \pm 8$. The mean shock reactivity and SEM for each group were as follows: sham, $968 \pm 75$; and hippocampus, $853 \pm 74$. Thus, differences in shock reactivity or contextual freezing during training cannot account for either the differences between groups in terms of contextual freezing during testing or the similarity in the enhancement of the startle reflex during testing.

\section{DISCUSSION}

The primary aim of these studies was to evaluate the role of the dorsal hippocampus in the expression of contextual fear condi- 
tioning using both freezing and fear-potentiated startle as measures of conditioned fear. In Experiment 1, we demonstrated that fear-potentiated startle, which has proven to be a valuable measure of fear to explicit cues, was also a reliable indicator of conditioned fear when used in a discriminable context conditioning procedure. In Experiment 2, we found that lesions of the central nucleus of the amygdala completely blocked both contextual freezing and fear-potentiated startle. Thus, the potentiation of startle we observe in our context conditioning procedure is both context-specific and amygdala-dependent. In Experiment 3, we found that post-training lesions of the dorsal hippocampus attenuated contextual freezing, consistent with previous reports in the literature (Kim and Fanselow, 1992; Phillips and LeDoux, 1992). Importantly, however, these same animals showed fearpotentiated startle comparable to that of sham-operated controls. This suggests that fear to the context was preserved in animals with hippocampal lesions, although the behavioral response of freezing was disrupted. It is unlikely that this elevation in startle in the hippocampal-lesioned group was an unconditioned effect of the lesion, because both the magnitude of the increase in startle and the time course of extinction during testing were comparable to those of the sham-operated controls. It is also unlikely that elevation in startle is attributable to a lack of habituation in the lesion group, because Leaton (1981) has demonstrated that rats with hippocampal lesions show normal short- and long-term habituation of the startle reflex.

One explanation for the present results would be that fearpotentiated startle had a lower response threshold than freezing. If this were the case, a lesion that disrupted but did not completely block fear could preferentially affect the freezing measure and not the startle measure. However, our data do not support this interpretation. In our first experiment, there was better discrimination between chambers with the startle measure than with the freezing measure. This suggests that freezing has a lower response threshold than fear-potentiated startle. Therefore one would be more, not less, likely to detect any disruptive effects of hippocampal lesions on contextual fear with the fear-potentiated startle measure than with the freezing measure.

Our interpretation of these results is that fear to the context is preserved in animals with hippocampal lesions, and that these lesions produce an unconditioned effect on activity that interferes with the expression of freezing. There is considerable evidence that supports this hypothesis. First, a number of studies have demonstrated that hippocampal lesions increase activity (Roberts et al., 1962; Teitelbaum and Milner, 1963; Douglas and Isaacson, 1964; Blanchard et al., 1977; Diaz-Granados et al., 1994; Good and Honey, 1997). Moreover, Maren and Fanselow (1997) have reported recently that the increase in open field activity produced by lesions of the hippocampus, entorhinal cortex, or fimbriafornix was significantly correlated with the disruption of contextual freezing observed in the same animals. Thus, it seems reasonable to speculate that hippocampal lesions might produce an unconditioned effect on activity that interferes with freezing.

The most obvious problem with the simple performance effect hypothesis is that one would expect freezing to explicit cues to be affected in the same manner as freezing to contextual cues. However, none of the studies comparing the effects of hippocampal lesions on contextual and explicit cue conditioning have equated the strength of conditioning. In these studies, the explicit cue is likely to have overshadowed the context, resulting in relatively strong freezing in the case of the explicit cue and relatively weak freezing in the case of the contextual cue. Even in the absence of an explicit cue, as in the present study, one would expect context conditioning to be weak, relative to the strength of conditioning that would accrue to an explicit conditioned stimulus (CS) given the same number of CS-unconditioned stimulus (US) pairings. Context is both less salient and a less valid predictor of reinforcement than an explicit $\mathrm{CS}$, two factors that determine the strength of conditioning (Rescorla and Wagner, 1972). Indeed, consistent with this expectation, Mast et al., (1982) demonstrated that an explicit CS elicited greater freezing than did contextual cues when the same number of signaled or unsignaled shocks had been given in training. Thus, hippocampal lesions may preferentially disrupt a weak freezing response but not a strong freezing response (Good and Honey, 1997). Furthermore, Maren et al., (1996) have recently reported that hippocampal lesions disrupt freezing to both explicit and contextual cues. Interestingly, the freezing to the explicit cues was disrupted less than the freezing to context, as one would expect if the disruptive effects of the lesions interacted with the strength of conditioning.

The most difficult results to account for with the present argument concern the retrograde time course of hippocampal lesions in disrupting contextual freezing reported by Kim and Fanselow (1992). They found that hippocampal lesions made either 1 or 7 but not $28 \mathrm{~d}$ after training disrupted contextual freezing but had no effect on freezing to the explicit cue. They concluded this was a direct measure of the importance of the hippocampus in the consolidation of contextual memories, as has been observed in the consolidation of declarative memories in humans and primates (Squire, 1992). Subsequently, Maren et al., (1996) have reported a similar disruption of freezing to both a contextual and explicit cue at 1 and 28 but not $100 \mathrm{~d}$ after training. The similar time course for the disruption of freezing to both the explicit cue and the context suggests a single process underlying both effects. An alternative interpretation of the above investigations is that they provided an indirect rather than direct measure of memory consolidation. If the memory for fear conditioning, both explicit and contextual, consolidates over time, it may become more resistant to the disruptive effects of hippocampal lesions in the same way that stronger conditioning is less disrupted by hippocampal lesions.

It is interesting that the first investigators to observe a deficit in freezing to a context paired previously with shock (Blanchard and Fial, 1968) came to a very similar conclusion to ours as to the nature of the deficit. After a series of investigations, they concluded that hippocampal lesions resulted in a subtle deficit in immobility (Blanchard et al., 1977). They showed that hippocampal-lesioned animals initiated freezing episodes as frequently as sham-lesioned animals, but these episodes were of shorter duration and resulted in less overall freezing. The deficit in immobility was more evident in the second half of the session than in the first, which supports the hypothesis that the lesion might interact with the strength of the conditioned freezing response. They, and others, observed freezing deficits in a variety of situations that presumably do not involve contextual representations, such as freezing in the presence of a predator (Kim et al., 1971; Blanchard and Blanchard, 1972), freezing to a shock probe, and freezing to prevent falling from a narrow ledge (Blanchard et al., 1977). In all cases, hippocampal-lesioned animals showed decreases in freezing relative to sham-operated controls.

There is also support for preserved contextual processing in animals with hippocampal lesions in behavioral paradigms that do not measure freezing. Bouton and colleagues have demonstrated 
that extinction, renewal, spontaneous recovery, and reinstatement of conditioned bar suppression are context dependent (Bouton and Bolles, 1979a,b; Bouton and King, 1983). Interestingly, Wilson et al. (1995) reported that rats with lesions of the fimbriafornix showed context specific extinction, renewal, and spontaneous recovery of conditioned bar suppression, suggesting preserved processing of the context, although there was a deficit in reinstatement. Similarly, Hall et al. (1996) reported that animals with hippocampal lesions showed no impairment in contextual control over bar pressing for a food reward.

A number of studies have demonstrated effects of hippocampal manipulations on spatial learning with no effect on contextual processing. Gallagher and Holland (1992) reported that rats with lesions of the hippocampus showed normal feature-positive and feature-negative discriminations, although these same animals were impaired on a spatial learning task. This finding demonstrated that rats with hippocampal lesions could make complex configural associations of which contexts are believed to be a special case. More recently, Good and Honey (1997) found that hippocampal and entorhinal cortex lesions disrupted spatial learning; however, only the hippocampal lesions disrupted contextual freezing. Importantly, the hippocampal lesions increased activity, whereas the entorhinal cortex lesions did not. Of particular interest is the recent study by Mayford et al. (1996), which allowed for both regional and temporal control over the expression of a calcium-independent form of calcium- and calmodulindependent kinase II (CaMKII) that was shown to disrupt longterm potentiation (LTP). They found that when the mutation was expressed in the hippocampus, it disrupted spatial learning on the Barnes circular maze but had no effect on the acquisition of contextual fear. In contrast, when the same transgene was expressed in the lateral nucleus of the amygdala during the acquisition of fear conditioning, it disrupted freezing to both the explicit cue and context in a test session 24 hr later. Thus, these data could be viewed as further evidence that the hippocampus, or at least CaMKII-dependent LTP in the hippocampus, is not necessary for contextual fear conditioning.

In conclusion, the present results, together with other reports in the literature, suggest that the hippocampus may not be critical for contextual fear conditioning. Moreover, they stress the importance of using multiple measures of conditioning to distinguish between the effects of a given experimental manipulation on a particular behavioral measure as opposed to the underlying phenomenon of interest.

\section{REFERENCES}

Blanchard RJ, Fial RA (1968) Effects of limbic lesions on passive avoidance and reactivity to shock. J Comp Physiol Psychol 66:606-612.

Blanchard RJ, Blanchard DC (1972) The effects of hippocampal lesions on the rat's reaction to a cat. J Comp Physiol Psychol 78:77-82.

Blanchard DC, Blanchard RJ, Lee MC, Fukunaga KK (1977) Movement arrest and the hippocampus. Physiol Psychol 5:331-335.

Bouton ME, Bolles RC (1979a) Contextual control of the extinction of conditioned fear. Learn Motiv 10:445-466.

Bouton ME, Bolles RC (1979b) Role of conditioned contextual stimuli in reinstatement of extinguished fear. J Exp Psychol Anim Behav Processes 5:368-378.

Bouton ME, King DA (1983) Contextual control of the extinction of conditioned fear: tests for the associative value of the context. J Exp Psycho Anim Behav Processes 9:248-265.

Brown JS, Kalish HI, Farber IE (1951) Conditioned fear as revealed by magnitude of startle response to an auditory stimulus. J Exp Psychol 41:317-328.

Campeau S, Davis M (1995) Involvement of the central nucleus and basolateral complex of the amygdala in fear conditioning measured with fear-potentiated startle in rats trained concurrently with auditory and visual conditioned stimuli. J Neurosci 15:2301-2311.

Campeau S, Hayward MD, Hope BT, Rosen JB, Nestler EJ, Davis M (1991) Induction of the c-fos proto-oncogene in the rat amygdala during unconditioned and conditioned fear. Brain Res 565:349-352.

Cassella JV, Davis M (1986) The design and calibration of a startle measurement system. Physiol Behav 36:377-383.

Davis M (1986) Pharmacological and anatomical analysis of fear conditioning using the fear-potentiated startle paradigm. Behav Neurosci 100:814-824.

Davis M (1992) The role of the amygdala in fear-potentiated startle: implications for animal models of anxiety. Trends Pharmacol Sci 13:35-41.

Diaz-Granados JL, Greene PL, Amsel A (1994) Selective activity enhancement and persistence in weanling rats after hippocampal Xirradiation in infancy: possible relevance for ADHD. Behav Neural Biol 61:251-259.

Douglas RJ (1967) The hippocampus and behavior. Psychol Bull 67:416-442.

Douglas RJ, Isaacson RL (1964) Hippocampal lesions and activity. Psychon Sci 1:187-188.

Gallagher M, Holland PC (1992) Preserved configural learning and spatial learning impairment in rats with hippocampal damage. Hippocampus 2:81-88.

Gewirtz JC, Falls WA, Davis M (1997) Normal conditioned inhibition and extinction of freezing and fear-potentiated startle following electrolytic lesions of medial prefrontal cortex in rats. Behav Neurosci 111:712-726.

Good M, Honey RC (1997) Dissociable effects of selective lesions to hippocampal subsystems on exploratory behavior, contextual learning, and spatial learning. Behav Neurosci 111:487-493.

Hall G, Purves D, Bonardi C (1996) Contextual control of conditioned responding in rats with dorsal hippocampal lesions. Behav Neurosci 110:933-945.

Hitchcock JM, Davis M (1986) Lesions of the amygdala, but not of the cerebellum or red nucleus, block conditioned fear as measured with the potentiated startle paradigm. Behav Neurosci 100:11-22.

Kapp BS, Gallagher M, Underwood MD, McNall CL, Whitehorn D (1982) Cardiovascular responses elicited by electrical stimulation of the amygdala central nucleus in the rabbit. Brain Res 234:251-262.

Kapp BS, Pascoe JP, Bixler MA (1984) The amygdala: a neuroanatomical systems approach to its contribution to aversive conditioning. In: The neuropsychology of memory (Butters N, Squire LS, eds), pp 473-488. New York: Guilford.

Kim JJ, Fanselow MS (1992) Modality-specific retrograde amnesia of fear. Science 256:675-677.

Kim C, Kim CC, Kim JK, Kim MS, Chang HK, Kim JY, Lee IG (1971) Fear response and aggressive behavior of hippocampectomized house rats. Brain Res 29:237-251.

Leaton RN (1981) Habituation of startle response, lick suppression, and exploratory behavior in rats with hippocampal lesions. J Comp Physiol Psychol 95:813-826.

Leaton RN, Borszcz GS (1985) Potentiated startle: its relation to freezing and shock intensity in rats. J Exp Psychol Anim Behav Process 11:421-428.

LeDoux JE (1992) Brain mechanisms of emotion and emotional learning. Curr Opin Neurobiol 2:191-198.

Maren S, Fanselow M (1997) Electrolytic lesions of the fimbria/fornix, dorsal hippocampus, or entorhinal cortex produce anterograde deficits in contextual fear conditioning in rats. Neurobiol Learn Mem 67:142-149.

Maren S, Aharonov G, Fanselow MS (1996) Excitotoxic dorsal hippocampal lesions and pavlovian fear conditioning in rats. Soc Neurosci Abstr 22:1379.

Mast M, Blanchard RJ, Blanchard DC (1982) The relationship of freezing and response suppression in a CER situation. Psychol Rec 32:151-167.

Mayford M, Bach ME, Huang YY, Wang L, Hawkins RD, Kandel ER (1996) Control of memory formation through regulated expression of a CaMKII transgene. Science 274:1678-1683.

Paxinos G, Watson C (1997) The rat brain in stereotaxic coordinates, Ed 3. San Diego: Academic. 
Phillips RG, LeDoux JE (1992) Differential contribution of amygdala and hippocampus to cued and contextual fear conditioning. Behav Neurosci 106:274-285.

Phillips RG, LeDoux JE (1994) Lesions of the dorsal hippocampal formation interfere with background but not foreground contextual fear conditioning. Learn Mem 1:34-44.

Rescorla RA, Wagner AR (1972) A theory of Pavlovian conditioning: variations in the effectiveness of reinforcement and nonreinforcement. In: Classical conditioning II: current research and theory (Black $\mathrm{AH}$ Prokasy WF, eds), pp 64-99. New York: Appleton-Century-Crofts.

Roberts WW, Dember WN, Brodwick M (1962) Alternation and exploration in rats with hippocampal lesions. J Comp Physiol Psychol 55:695-700.
Sananes CB, Davis M (1992) N-Methyl-D-aspartate lesions of the lateral and basolateral nuclei of the amygdala block fear-potentiated startle and shock sensitization of startle. Behav Neurosci 106:72-80.

Squire LR (1992) Memory and the hippocampus: a synthesis from findings with rats, monkeys, and humans. Psychol Rev 99:195-231.

Teitelbaum H, Milner P (1963) Activity changes following partial hippocampal lesions in rats. J Comp Physiol Psychol 56:281-289.

Wagner AR (1981) SOP: a model of automatic memory processing in animal behavior. In: Information processing in animals: memory mechanisms (Spear NE, Miller RR, eds), pp 5-47. Hillsdale, NJ: Erlbaum.

Wilson A, Brooks DC, Bouton ME (1995) The role of the rat hippocampal system in several effects of context in extinction. Behav Neurosci 109:828-836. 